\title{
Impact of COVID19 pandemic on the incidence of health-care associated Clostridioides difficile infection
}

Nicolás Merchante ( $\nabla$ nicolasmerchante@gmail.com )

Unidad de Enfermedades Infecciosas y Microbiología. Hospital Universitario de Valme, Universidad de Sevilla

\section{Pablo Chico}

Hospital General Universitario de Alicante

\section{Esther Márquez-Saavedra}

Hospital Universitario de Valme

\section{Gerónima Riera}

Hospital General Universitario de Alicante

\section{Rocío Herrero}

Unidad de Enfermedades Infecciosas y Microbiología. Hospital Universitario de Valme

\section{Pilar González-de-la-Aleja}

Hospital General Universitario de Alicante

\section{Ana I. Aller}

Unidad de Enfermedades Infecciosas y Microbiología. Hospital Universitario de Valme Juan Carlos Rodríguez

Hospital General Universitario de Alicante

\section{Miguel Rodríguez-Fernández}

Unidad de Enfermedades Infecciosas y Microbiología. Hospital Universitario de Valme José Manuel Ramos

Hospital General Universitario de Alicante

\section{Marta Trigo-Rodríguez}

Unidad de Enfermedades Infecciosas y Microbiología. Hospital Universitario de Valme

\section{Esperanza Merino}

Hospital General Universitario de Alicante

\section{Research Article}

Keywords: SARS-CoV-2, Covid-19, Clostridioides difficile, Health-care associated infections, Antimicrobial stewardship 
Posted Date: February 1st, 2022

DOI: https://doi.org/10.21203/rs.3.rs-1304155/v1

License: (c) (i) This work is licensed under a Creative Commons Attribution 4.0 International License. Read Full License 


\section{Abstract}

Objective: To investigate the impact of COVID19 pandemic on the incidence of health-care associated Clostridioides difficile infection (HA-CDI).

Methods: Retrospective study conducted in the Hospital Universitario de Valme (HUV) and the Hospital General Universitario de Alicante (HGUA) in Spain between January 2019 and February 2021. The study period was divided into non-COVID19 period (2019 and months from 2020-2021 with $\leq 30$ hospitalized COVID19 patients) and COVID19 period (months from 2020-2021 with > 30 COVID19 patients). HA-CDI incidence rates (IR) were calculated as the number of new CDI cases per 10.000 occupied bed-days (OBD) and antimicrobial consumption by means of the defined daily dose (DDD) per 1000 OBD.

Results: During the COVID19 period, HA-CDI IR in the HUV was 2.6 per 10.000 OBD, which was lower than what was observed during the non-COVID19 period (4.1 per 10.000 OBD; $p=0.1$ ). In the HGUA, HA-CDI IR during COVID19 period was 3.9 per 10.000 OBD, which was not significantly different to the IR observed during the non-COVID19 period (3.7 per 10.000 OBD; $p=0.8$ ). There was a slight increase in the total antibiotic consumption during COVID19 period in both hospitals, with significant increases of certain high-risk antibiotics as cephalosporins.

Conclusions: HA-CDI incidence has not increased during the COVID19 pandemic in two tertiary centers in Spain, in spite of a slightly higher antibiotic consumption during the COVID19 period in both hospitals. These findings suggest that, in the presence of strict infection control measures, hospital antibiotic consumption might have a lower impact than expected on HA-CDI.

\section{Key Points}

- Healthcare-associated CDI incidence has not increase during COVID19 in spite of higher antibiotic consumption.

- Decreases of hospital-onset healthcare facility-associated CDI incidence in every wave were seen in one center.

- Strict infection control measures mitigate the impact of hospital antibiotic use.

\section{Background}

Clostridioides difficile is the leading cause of nosocomial infectious diarrhea and one of the most prevalent health-care associated infections (HAls) [1,2]. Besides patient exposure to C.difficile spores colonizing the environment, the leading predisposing factors for $C$.difficile colonization and infection are, among others, previous exposure to systemic antibiotics and a variety of comorbidities, including immunosuppression. ${ }^{3}$ Consequently, environment cleaning, hand hygiene and antibiotic restriction have been the most widely accepted interventions for $C$.difficile infection (CDI) prevention [4]. 
Coronavirus disease 2019 (COVID19) pandemic have placed an enormously demand on the health care system. The high rates of COVID19 admissions have led to the adoption of extraordinary organizational measures in acute care hospitals, which have been common across different countries, such as the creation of COVID medical wards where patients were under isolation measures and health-care workers wore personal protective equipment (PPE). In fact, increased attention to infection prevention practices, including hand hygiene and contact precautions compliance, has been an undoubtedly collateral effect of the COVID19 pandemic. Theoretically, this enhancement of infection control measures might have impact in the spread of multi-drug resistant organisms (MDROs) or C.difficile within hospitals. However, other collateral effects of the pandemic, such as the shift of resources for the monitoring and prevention of HAls to support the COVID19 response and the discontinuation of antimicrobial stewardship (AMS) activities, could have act in the opposite direction, leading to high HAls, including CDI.

The impact of COVID19 pandemic on the incidence of CDI has been assessed in a few studies with conflicting results. Although some experts have speculated that hospitalized patients with COVID19 could be more prone to develop CDI [5], most of the studies conducted so far have found either no effect [6-8] or a reduction of $C D I$ incidence during the first wave of the pandemic [9-12]. A common limitation of these studies is that they have focused only in the first months of the pandemic. However, the impact of COVID19 on CDI incidence might be affected by the evolution of several factors during the pandemic. First, medical management of hospitalized patients with COVID19 have largely evolved, including the widely use of corticosteroids and other immunosuppressive agents. Second, the profile of hospitalized patients has also changed, with less comorbidities and younger ages. Finally, while aggressive lockdowns and the stop of surgeries and medical attention to non-COVID conditions was a constant among most countries during the first wave, a progressive coexistence of COVID19 and non-COVID19 medical care within hospitals have been adopted in the subsequent months of the pandemic.

For the above-mentioned reasons, our objective was to investigate the impact of the first year of COVID19 pandemic on the incidence of health-care associated CDI (HA-CDI).

\section{Methods}

\section{Study design, study periods and sources of data}

The ICD-ANCRAID-SEICV cohort (ClinicalTrials.gov ID: NCT04801862) is a prospective multicentric cohort recruiting all consecutive patients with a new diagnosis of CDI from 15 hospitals from Spain. This was a retrospective study conducted in two tertiary care hospitals (Hospital General Universitario de Alicante [HGUA], n=783 beds; Hospital Universitario de Valme [HUV], $n=581$ beds) from the cohort between January 2019 and February 2021.

The study period was divided into non-COVID19 period (2019 and months from 2020-2021 with 30 or less patients hospitalized due to COVID19) and COVID19 period (months from 2020-2021 with > 30 COVID19 patients). The threshold of 30 patients for was decided by the investigators as an indirect indicator of a 
monthly occupancy bed rate due to COVID19 $>5 \%$. For this purpose, we considered only those admissions due to clinical symptoms related to SARS-CoV-2 infection that was microbiologically confirmed by polymerase chain reaction (PCR) or an antigen test.

All CDI episodes diagnosed in patients older than 18 years in the participant hospitals during the study period were extracted by from specific databases from the Infectious Diseases Units that were also checked with microbiology laboratory reports and Preventive Medicine registries. The electronic clinical records of all CDI cases were reviewed for this study in order to confirm that fulfilled required criteria for $\mathrm{CDI}$ diagnosis and health-care associated origin (see Definitions on the next section).

\section{Definitions and microbiological procedures}

CDI diagnosis was established following the European Centre for Disease Prevention and Control (ECDC) [13] and the European Society of Clinical Microbiology and Infectious Diseases (ESCMID) recommendations $[14,15]$ by the presence of diarrhea and a positive laboratory assay for $C$.difficiletoxin $A$ and/or B in stools or a toxin-producing C.difficileorganism detected in stool by means of a polymerase chain reaction (PCR) method. According to ECDC criteria, a CDI episode was classified as HA-CDI if the onset of symptoms occurred on day three or later following admission to a healthcare facility or within four weeks of discharge from a healthcare facility [13]. We also analyzed CDI cases considering only hospital-onset healthcare facility-associated (HO-HCFA) as defined by the Infectious Diseases Society of America (IDSA) [16]. CDI incidence rates (IR) were calculated as the number of new CDI cases per 10.000 occupied bed-days (OBD).

The algorithm employed for the microbiological diagnosis of CDI did not changed throughout the study period and included a sequential qualitative detection of glutamate dehydrogenase and toxin $B$ from $C$. difficile by immunochromatography (HUV: CerTest, BIOTEC S.L., Spain; HGUA: C. difficile Quik Chek complete, TechLab, Blacksburg, VA). We assessed discrepancies by detecting the $C$. difficile toxin $B$ gene by real-time PCR (HUV: BD MAX Cdiff, Becton Dickinson Diagnostics, Canada; HGUA: GeneXpert C. difficile/Epi test, Cepheid, Sunnyvale, California). The number of stool samples sent to microbiology for $C$. difficile diagnosis standardized per 10.000 OBD during the study periods was also assessed.

\section{Antimicrobial consumption}

Antimicrobial consumption was assessed by means of the calculation of the defined daily dose (DDD) per 1000 OBD, according to the Anatomical Therapeutic Chemical Classification System (ATC/DDD) defined by WHO [17].Total antimicrobial consumption (ATC codes J01 and J02) and the consumption of specific antimicrobial families which has been linked to an increased risk of CDI (penicillins, cephalosporins, carbapenems, piperacillin/tazobactam, fluoroquinolones and clindamycin) $[18,19]$ were evaluated. 


\section{Infection control measures}

A similar infection prevention bundle was implemented during the COVID19 period in both participant hospitals. Educational activities with special emphasis on hand hygiene and infection control measures were developed to health-care workers. All healthcare workers wore PPE when caring for patients with COVID19 whereas the universal use of hand gloves when attending non-COVID19 patients was discouraged. Environmental cleaning by trained cleaning staff was reinforced during the COVID19 period but the same cleaning products were employed for both periods. Patients with COVID-19 were grouped in COVID-wards ( 2 or 3 patients per room) with dedicated staff. Visits were prohibited to COVID19 wards (except in exceptional situations) and restricted to non-COVID19 wards during the COVID19 period. Elective procedures and non-urgent admissions were suspended several times during the COVID19 pandemic depending on hospitals occupancy rates. Patients with CDI were isolated in a single room with contact precautions during both the COVID-19 and non-COVID19 periods.

\section{Statistical analyses}

Continuous variables were expressed as median (Q1-Q3) and categorical variables as frequencies (percentage). Continuous variables were compared by means of the Student $t$ test or the Mann-Whitney $U$ test, depending on the normality tests. The chi-square and the Fisher tests were used for comparisons between categorical variables.

HA-CDI IR and its respective 95\% confidence intervals $(\mathrm{Cl})$ during the study periods in each participant hospital were computed. HA-CDI IR comparisons between COVID-19 and non COVID-19 periods were performed by calculating the rate ratio (RR) with its respective $95 \% \mathrm{Cl}$. Associations with a $p<0.05$ were considered significant. Analyses were performed with SPSS statistics (version 25; IBM Corp., Armonk, NY).

\section{Results}

Since March 2020, when the first COVID19 patients were admitted in the HUV and the HGUA, the evolution of the number of hospitalized patients due to COVID19 is presented in Figure 1. Reflecting the different evolution of the pandemic among the different regions in Spain, the duration and the intensity of consecutive waves of the epidemic was different between the 2 participant hospitals. In HUV the COVID19 period included 3 well defined different waves (Figure 1A), whereas in HGUA an overlap between $2^{\text {nd }}$ and $3^{\text {rd }}$ waves was seen (Figure $1 B$ ).

\section{Hospital Universitario de Valme}

\section{Impact of COVID19 on CDI incidence}


During the COVID19 period, 19 HA-CDI episodes were diagnosed, leading to a CDI incidence of 2.6 per 10.000 OBD, which was lower than what was observed during the non-COVID19 period ( $n=86 \mathrm{HCA}-\mathrm{CDI}$ cases; $C D I$ incidence 4.04 per $10.000 \mathrm{OBD})(p=0.1)$ (Table 1). Figure $2 \mathrm{~A}$ shows the monthly distribution of HA-CDI incidence and total antibiotic consumption in HUV during the study period. Of note, a marked decreased in HA-CDI incidence in every COVID19 wave was noted. This behavior was even more apparent when only HO-HCFA cases were included (Figure 3). During 2019, 977 CDI diagnostic test were performed, which accounted for 71.5 per 10.000 OBD, whereas 1022 test were done in 2020 accounting for 80.7 per $10.000 \mathrm{OBD}(\mathrm{p}=0.08)$. The mean number of CDI diagnostic test performed per 10.000 OBD during nonCOVID19 period was 77.7 whereas in COVID19 period was $67.1(p=0.04)$.

\section{Impact of COVID on antimicrobial consumption}

There were no differences between the total antibiotic consumption between the non-COVID 19 and the COVID19 period (518 vs 543 DDD per 1000 OBD; $p=0.3$ ) (Figure 3). However, when analyzing specific antibiotic classes, there were significant increases in the consumption of certain high-risk antibiotics as cephalosporins and carbapenems, although overall consumption of high-risk antibiotics was comparable in both periods (Figure 4A). Remarkably, a $22 \%$ increase in the consumption of cephalosporins was seen during the COVID19 period.

\section{Hospital General Universitario de Alicante}

\section{Impact of COVID19 on CDI incidence}

During the COVID19 period, $56 \mathrm{HA}-\mathrm{CDI}$ episodes were diagnosed, leading to a CDI incidence of 3.9 per 10.000 OBD, which was not significantly different to what was observed during the non-COVID19 period $(n=100$ HCA-CDI cases; $C D I$ incidence 3.7 per 10.000 OBD) $(p=0.8)$ (Table 1). The monthly distribution of HA-CDI incidence and total antibiotic consumption in the HGUA during the study period is shown in Figure 2B. CDI diagnostic test were performed in 89.8 per 10.000 OBD in 2019 and in 92.2 per 10.000 OBD in $2020(p=0.5)$. The mean number of CDI diagnostic test performed per 10.000 OBD during non-COVID19 period was 89.2 whereas in COVID19 period was $93.1(p=0.4)$.

Table 1

Incidence rates of health-care associated Clostridioides difficile infection according to study periods in each participant center. 


\begin{tabular}{|c|c|c|c|c|}
\hline Category & $\begin{array}{l}\text { No. of HA- } \\
\text { CDI }^{1} \\
\text { episodes }\end{array}$ & $\begin{array}{l}\text { Occupied } \\
\text { bed days }\end{array}$ & $\begin{array}{l}\text { Incidence }{ }^{2} \text { and incidence rate } \\
\text { ratio } \\
\left(95 \% \mathrm{Cl}^{3}\right)\end{array}$ & $\mathbf{p}$ \\
\hline \multicolumn{4}{|l|}{ Global $\left(H^{4} V^{4}+H_{G U A}^{5}\right)$} & \multirow[t]{4}{*}{0.5} \\
\hline Non-COVID19 period & 186 & 478076 & 3.89 & \\
\hline COVID19 & 75 & 213239 & 3.52 & \\
\hline \multicolumn{2}{|l|}{$\begin{array}{l}H A-I C D^{1} \text { incidence rate } \\
\text { ratio }\end{array}$} & & $0.90(0.69-1.18)$ & \\
\hline \multicolumn{2}{|l|}{ HUV 4} & & & \multirow[t]{4}{*}{0.1} \\
\hline Non-COVID19 period & 86 & 212616 & 4.04 & \\
\hline COVID19 & 19 & 73016 & 2.6 & \\
\hline \multicolumn{2}{|l|}{$\begin{array}{l}H A-I C D^{1} \text { incidence rate } \\
\text { ratio }\end{array}$} & & $0.64(0.39-1.06)$ & \\
\hline \multicolumn{2}{|l|}{ HGUA $^{5}$} & & & \multirow[t]{2}{*}{0.8} \\
\hline Non-COVID19 period & 100 & 265460 & 3.77 & \\
\hline COVID19 period & 56 & 140223 & 3.99 & \\
\hline \multicolumn{2}{|l|}{$\begin{array}{l}H A-I C D^{1} \text { incidence rate } \\
\text { ratio }\end{array}$} & & $1.06(0.76-1.47)$ & \\
\hline \multicolumn{5}{|c|}{$\begin{array}{l}{ }^{1} \mathrm{HA}-\mathrm{ICD} \text { : Health-care associated clostridoides difficile infection; }{ }^{2} \text { Incidence are expressed per } 10.000 \\
\text { OBD; }{ }^{3} \mathrm{Cl} \text { : Confidence interval; }{ }^{4} \mathrm{HUV} \text { : Hospital Universitario de Valme; }{ }^{5} \mathrm{HGUA} \text { : Hospital General } \\
\text { Universitario de Alicante. The study period was divided into non-COVVID } 19 \text { period ( } 2019 \text { and months } \\
\text { from } 2020-2021 \text { with } 30 \text { or less patients hospitalized due to COVID19) and COVID } 19 \text { period (months } \\
\text { from } 2020-2021 \text { with > } 30 \text { COVID } 19 \text { patients). }\end{array}$} \\
\hline
\end{tabular}

In HGUA, a COVID19 unit with a specific informatic code of admission was created. Thus, specific COVID19 OBD and the incidence of HA-CDI in the COVID19 unit could be computed. Using this approach, 7 cases of CDI among 17347 OBD (HCA-CDI incidence: 4.03 per 10.000 OBD) were detected in the COVID19 unit whereas 145 among 373290 OBD (HA-CDI incidence: 3.88 per 10.000 OBD) were diagnosed in non-COVID19 units since from 2019 to February 2021. HA-CDI incidence in non-COVID19 units did not change when it was calculated considering only the pandemic period (March 2020-February 2021; $n=70$ HA-CDI cases; HA-CDI incidence 3.8 per 10.000 OBD).

\section{Impact of COVID on antimicrobial consumption}


There was a non-significant slight increase in the total antibiotic consumption between the non-COVID 19 and the COVID19 period (874 vs 984 per 1000 OBD; $p=0.3$ ) (Figure 4B). A significant increase of the consumption of some high-risk antibiotics, such as cephalosporins (which increased by a factor of $41 \%$ ) and piperacillin/tazobactam, was seen in the COVID19 period whereas the consumption of quinolones decreased (Figure 4B).

\section{Discussion}

Our study shows that HA-CDI incidence has not increased during the COVID19 pandemic in two tertiary centers in Spain. We have also observed that the evolution of HA-CDI has been heterogeneous between the two participant centers. Thus, a significant decrease of HA-CDI incidence in every wave of the pandemic was observed in the HUV whereas incidence remained stable through the pandemic in HGUA. Interestingly, these figures were observed in the presence of a slightly higher antibiotic consumption during the COVID19 period in both hospitals, suggesting that, in the presence of strict infection control measures, hospital antibiotic consumption might have a lower impact than expected on HA-CDI.

At the beginning of the pandemic, some experts expressed concern that COVID19 could impact CDI rates, especially in the elderly [5]. However, initial studies have reported no impact or a trend for a lower incidence that anticipated by historical data. In the first study assessing this issue, Ponce-Alonso et al. reported a marked decrease in HO-HCFA incidence during the first wave in Madrid when compared to a historical control period in spite of a mild increase of antibiotics consumption [9]. A similar effect has been observed during the first wave in Dublin [10], Rome [11] and Mexico [12]. On the other hand, studies conducted during the first wave in New York [6] and Singapore [7] have found no effect of the pandemic on the incidence of CDI. Recently, Baker and coworkers did not found variations in HA-CDI incidence with respect to monthly COVID19 hospitalization rates during 2020 among 148 HCA Healthcare-affiliated hospitals in USA [8].

In the line with these previous reports, our work demonstrates that COVID19 has not promoted an increase in HA-CDI incidence during the first year of the pandemic. Of note, a marked decrease of HA-CDI during the first three waves of the pandemic was seen in the HUV in a similar manner to what has been seen in the first wave in other centers [9-12]. This behavior was even more pronounced when only HOHCFA cases were considered. These results suggest that, at least in some centers, the implementation of infection control measures and the reorganization of health-care activities during the pandemic have led to collateral beneficial effects in terms of Clostridioides difficile nosocomial transmission.

Antimicrobial exposure is one of most relevant individual factors associated with CDI development $[3,18,19]$. Besides direct patient exposure to antibiotics, the influence of antibiotic use on the risk of CDI may also operate at aggregate levels (i.e. ward or hospital use of antibiotics). In Scotland, limiting hospital use of high-risk antibiotics was associated with a substantial decline in CDI, especially those caused by epidemic ribotypes [20]. Recent studies conducted in the US have shown that facilities achieving significant reductions in hospital antibiotic use experienced reductions of HO-HCFA CDI, which 
was more evident for reductions in quinolones and cephalosporins [21,22]. By contrast, a lack of effect on CDI has been seen after 3 years of starting the PIRASOA program, an antimicrobial stewardship program implemented in hospitals of the Public Health System of Andalusia, Spain [23]. While PIRASOA has led to a significant decrease in total antimicrobial consumption and a reduction of the incidence of infections due MDROs in the Andalusian public health-care system, the incidence of CDI increased during the same period [23]. Likewise, other studies have not found a correlation between monthly cephalosporin hospital use and the risk of CDI [24].

In our study, a stable or declining incidence of HA-CDI was seen in the presence of a mild increase of total antibiotic consumption during the pandemic, including significant increases in certain high-risk antibiotics such as cephalosporins. Similarly, some of the previous studies reporting a decrease of CDI incidence during the first wave of the pandemic also found a concomitant increase of antibiotic consumption [6,9]. These data support that the impact of hospital antibiotic use on CDI rates is not uniform and depends on several factors, such as the degree of implementation of infection control measures, the prevalence of high-risk ribotypes or the level of use of specific antibiotic classes such as quinolones. In this sense, a "saturation effect" has been described for quinolones, as CDI risk rises until reaching a certain threshold of use beyond which risk plateaus [21]. In the line of this, we speculate that the heterogeneous effect of COVID19 between the two participant hospitals observed herein might be explained by different baseline levels of hospital antibiotic pressure. Thus, the lower baseline antibiotic pressure might have facilitated the observed decrease in CDI incidence during COVID19 in the HUV, whereas this was not seen in the HGUA as a consequence of a higher baseline antibiotic consumption and a marked increase of cephalosporin use during COVID19. While previous studies have suggested that reductions in CDI incidence can be expected in institutions with high-level of antibiotic consumption $[21,22]$, it is possible that mild changes in antibiotic use in settings where low to intermediate level of consumption has already been achieved might no further impact on CDI.

Our study has some limitations that must be acknowledged. First, as our study was observational and retrospective, definitive causal relationships cannot be inferred from our observations. Second, variations in $\mathrm{CDI}$ rates could be explained by under diagnosis due to the exceptional situation within hospitals during the COVID19 pandemic. We found this unlikely, as the standardized number of stool samples sent to the laboratory during the pandemic was comparable to the previous year. As a slight decrease in CDI diagnostic test during the COVID19 period was observed in the HUV, we cannot completely exclude that this could partially affect HA-CDI incidence rates. However, it would not justify the marked reductions of HO-HCFA rates that we have observed. Third, the direct effect of SARS-CoV2 infection on CDI risk in an individual basis could only be assessed in the HGUA, as in the HUV we could only assess monthly incidence of HA-CDI but not the incidence of CDI among hospitalized COVID19 patients. Finally, we compared antibiotic consumption between COVID19 and non COVID19 periods by means of DDD per 1000 OBD calculation but we could not evaluate if durations of antibiotic regimens differ between both periods, which would have been of interest, as the duration of antibiotic exposure has been linked to CDI risk [18]. On the other hand, previous studies have focused only in HO-HCFA CDI, which could 
underestimate the impact of COVID19 hospitalization in health-care associated CDI, as many of hospital acquired CDI cases become apparent after hospital discharge. In our study, we have included incident CDI cases up to 4 weeks after previous admission, which gives a better picture of the impact of the pandemic on HA-CDI. Besides, previous studies have evaluated the overall consumption of antibiotics during the pandemic without distinguishing between different classes. We have compared overall consumption during COVID19 but also by antibiotic families, as CDI risk is not uniform across them. Finally, changes in HA-CDI incidence is highly influenced by clinical suspicion and microbiological detection. To solve this, our analysis has also included standardized data of stool samples sent to microbiology during COVID19 and non-COVID19 periods. These are strengths of our study.

\section{Conclusion}

In summary, the COVID19 pandemic has not promote an increase on HA-CDI. The role of hospital consumption of antibiotics on the risk of HA-CDI might be less prominent than previously believed, at least in the presence of strict control measures. Consequently, public health and preventive strategies should still focus on other relevant factors such as environmental cleaning and safe-practices implementation.

\section{Declarations}

\section{Ethical aspects}

The study was designed and conducted following the Helsinki declaration. The ICD-ANCRAID-SEICV cohort (ClinicalTrials.gov ID: NCT04801862) protocol has been approved by the ethics committee of the Hospital Universitario de Valme.

\section{Consent for publication}

Not applicable

\section{Availability of data and materials}

The datasets used and/or analysed during the current study are available from the corresponding author on reasonable request

\section{Competing interest}

The authors declare that they have no competing interests. 


\section{Conflict of interest}

The authors have no conflict of interest to declare.

\section{Funding}

This work has been partially financed by the Instituto de Salud Carlos III (project PI20 / P1450) and cofinanced by the European Union (ERDF) "A way of making Europe". In addition, it has also been partially funded by Merck, Sharp and Dohme (Ref. IISP 60379 and 60386), including support for medical writing, but which had no role on its design, analysis or the decision to submit the results.

\section{Author contributions}

NM and EM had full access and to all the data in the study and takes responsibility for the integrity of the data and the accuracy of the data analysis.

Study concept and design: NM and EM.

Acquisition, analysis, or interpretation of data: NM, PC, EMS, GR, RH, PGA, AIA, JCR, MR, JMR, MTR, EM.

Statistical analysis: NM and PC.

Drafting of the manuscript: NM.

Critical revision of the manuscript for important intellectual content: NM, PC, EMS, GR, RH, PGA, AIA, JCR, MR, JMR, MTR, EM.

Obtained funding: NM.

Study supervision: NM and EM.

All authors have read and approved the manuscript.

\section{Acknowledgments}

Not applicable.

\section{References}

1. Lessa FC, Mu Y, Bamberg WM, Beldavs ZG, Dumyati G, Dunn JR, et al. Burden of Clostridium difficile infection in the United States. N Engl J Med. 2015;372:825-4. doi: 10.1056/NEJMoa1408913. 
2. Davies KA, Longshaw CM, Davis GL, Bouza E, Barbut F, Barna Z, et al. Underdiagnosis of Clostridium difficile across Europe: the European, multicenter, prospective, biannual, point-prevalence study of Clostridium difficile infection in hospitalized patients with diarrhea (EUCLID). Lancet Infect Dis. 2014;14:1208-19. doi: 10.1016/S1473-3099(14)70991-0.

3. Longo DL, Leffler DA, Lamont JT. Clostridium difficile infection. N Engl J Med. 2015;372:1539-48. doi: 10.1056/NEJMra1403772

4. Tschudin-Sutter S, Kuijper EJ, Durovic A, Vehreschild MJGT, Barbut F, Eckert C, et al. Guidance document for prevention of Clostridium difficile infection in acute healthcare settings. Clin Microb Infect. 2018;24:1051-4. doi: 10.1016/j.cmi.2018.02.020. Epub 2018 Mar 2.

5. Spigaglia P. COVID-19 and Clostridioides difficile infection (CDI): Possible implications for elderly patients. Anaerobe. 2020; 64:102233. doi: 10.1016/j.anaerobe.2020.102233.

6. Luo Y, Grinspan LT, Fu Y, Adams-Sommer V, Willey DK, Patel G, et al. Hospital-onset Clostridoides difficile infections during the COVID-19 pandemic. Infect Control Hosp Epidemiol. 2021;42:1165-6. doi: $10.1017 /$ ice.2020.1223

7. Wee LEI, Conceicao EP, Tan JY, Magesparan KD, Amin IBM, Ismail BBS, et al. Unintended consequences of infection prevention and control measures during COVID-19 pandemic. Am J Infect Control. 2021;49:469-77. doi: 10.1016/j.ajic.2020.10.019.

8. Baker MA, Sands KE, Huang SS, Kleinman K, Septimus EJ, Varma N, et al. The impact of COVID-19 on healthcare-associated infections. Clin Infect Dis. 2021; Aug9; ciab688. doi: 10.1093/cid/ciab688. Online ahead of print. doi: 10.1093/cid/ciab688.

9. Ponce-Alonso M, Sáez de la Fuente J, Rincón-Carlavilla A, Moreno-Nuñez P, Martínez-García L, Escudero-Sánchez R, et al. Impact of the coronavirus disease 2019 (COVID-19) pandemic on nosocomial Clostridioides difficile infection. Infect Control Hosp Epidemiol. 2021;42:406-10. doi: 10.1017/ice.2020.454.

10. Hazel K, Skally M, Glynn E, Foley M, Burns K, O'Toole A, et al. The other 'C': Hospital-acquired Clostridioides difficile infection during coronavirus disease 2019 (COVID-19) pandemic. Infect Control Hosp Epidemiol. 2021 Jan 13:1-2. doi: 10.1017/ice.2021.3. Online ahead of print. doi: 10.1017/ice.2021.3

11. Bentivegna E, Alessio G, Spuntarelli V, Luciani M, Santino I, Simmaco M, et al. Impact of COVID-19 prevention measures on risk of health care-associated Clostridium difficile infection. Am J Infect Control. 2021;49:640-2. doi: 10.1016/j.ajic.2020.09.010.

12. Ochoa-Hein E, Rajme-López S, Rodríguez-Aldama JC, Huertas-Jiménez MA, Chávez-Ríos AR, PazGarcía P, et al. Substantial reduction of healthcare facility-onset Clostridoides difficile infection (HO$\mathrm{CDI}$ ) rates after conversion of a hospital for exclusive treatment of COVID-19 patients. Am J Infect Control. 2021;49:966-8. doi: 10.1016/j.ajic.2020.12.008.

13. European Centre for Disease Prevention and Control. European surveillance of Clostridioides (Clostridium) difficile infections. Surveillance protocol version 2.4. Stockholm: ECDC; 2019. doi 
10.2900/60304. Available at: ecdc.europa.eu/sites/default/files/documents/clostridium-difficileinfections-EU-surveillance-protocol-vers2.4.pdf

14. Crobach MJ, Planche T, Eckert C, Barbut F, Terveer EM, Dekkers OM, et al. European Society of Clinical Microbiology and Infectious Diseases: update of the diagnostic guidance document for Clostridium difficile infection. Clin Microbiol Infect. 2016;22:S63-81. doi: 10.1016/j.cmi.2016.03.010.

15. van Prehn J, Reigadas E, Vogelzang EH, Bouza E, Hristea A, Guery B, et al. European Society of Clinical Microbiology and Infectious Diseases: 2021 update on the treatment guidance document for Clostridioides difficile infection in adults. Clin Microbiol Infect. 2021 Oct 19;S1198-743X(21)005681.doi:10.1016/j.cmi.2021.09.038. Online ahead of print.

16. Mc Donald CL, Gerding DN, Johnson S, Bakken JS, Carrol KC, Coffin SE, et al. Clinical practice guidelines for Clostridium difficile infection in adults and children: 2017 update by the Infectious Diseases Society of America (IDSA) and Society for Healthcare Epidemiology of America (SHEA). Clin Infect Dis. 2018;66:987-94. doi: 10.1093/cid/ciy149.

17. World Health Organization Collaborating Center for Drug Statistics Method- ology, Norwegian Institute of Public Health. Definition and general considerations: defined daily dose (DDD). 2018. Available at: https://www.whocc. no/ddd/definition_and_general_considera/.

18. Brown KA, Langford B, Schwartz KL, Diong C, Garber G, Daneman N. Antibiotic prescribing choices and their comparative $C$.difficile infection risks: a longitudinal case-cohort study. Clin Infect Dis. 2021;72:836-44. doi: 10.1093/cid/ciaa124.

19. Webb BJ, Subramanian A, Lopansri B, Goodman B, Jones PB, Ferraro J, et al. Antibiotic exposure and risk for hospital-associated Clostridioides difficile infection. Antimicrob Agents Chemother. 2020;64:e01269-19. doi: 10.1128/AAC.02169-19.

20. Lawes T, Lopez-Lozano JM, Nebot CA, Macartney G, Subbarao-Sharma R, Wares KD, et al. Effect of a national $4 \mathrm{C}$ antibiotic stewardship intervention on the clinical and molecular epidemiology of Clostridium difficile infections in a region of Scotland: a non-linear time-series analysis. Lancet Infect Dis. 2017;17:194-206. doi: 10.1016/S1473-3099(16)30397-8.

21. Kazakova SV, Baggs J, Mc Donald LC, Yi SH, Hatfield KM, Guh A, et al. Association between antibiotic use and hospital-onset Clostridioides difficile infection in US acute care hospitals, 2006-2012: An ecologic analysis. Clin Infect Dis. 2020;70:11-8. doi: 10.1093/cid/ciz169.

22. Kazakova SV, Baggs J, Yi SH, Reddy SC, Hatfield KM, Guh AY, et al. Associations of facility-level antibiotic use and hospital-onset Clostridioides difficile infection in US acute-care hospitals, 20122018. Infect Control Hosp Epidemiol. 2021;7:1-3. doi: 10.1017/ice.2021.151.

23. Rodriguez-Baño J, Pérez-Moreno MA, Peñalva G, Garnacho-Montero J, Pinto C, Salcedo I, et al. Outcomes of the PIRASOA programme, an antimicrobial stewardship programme implemented in hospitals of the Public Health System of Andalusia, Spain: an ecologic study of time-trend analysis. Clin Microbiol Infect. 2020;26:358-65. doi: 10.1016/j.cmi.2019.07.009.

24. Kim B, Kim J, Pai H. Association between antibiotic consumption and incidence of Clostridioides difficile infection in a hospital. J Korean Med Sci. 2020;35(47):e407. doi: 


\section{Figures}

$1 \mathrm{~A}$

Evolution of the number of hospitalized COVID19 patients in HUV

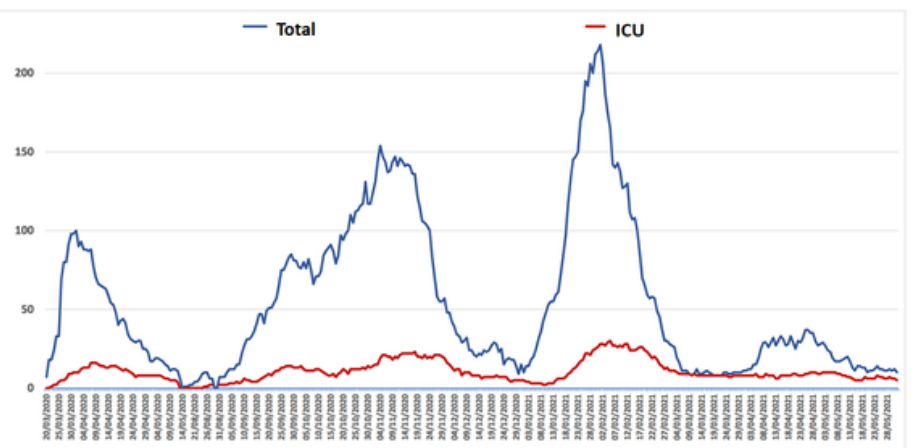

1B Evolution of the number of hospitalized COVID19 patients in HGUA

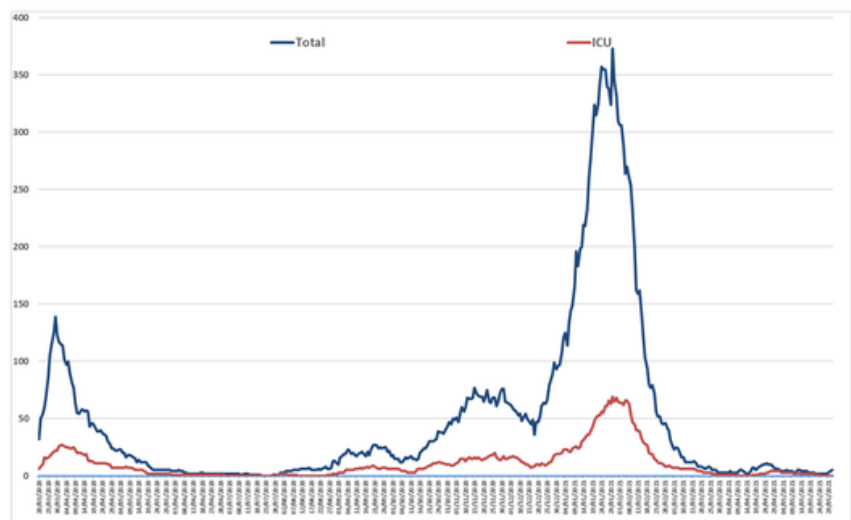

Figure 1

Evolution of the number of hospitalized patients due to COVID19 in the participant hospitals. 1A: Hospital Universitario de Valme (HUV). 1B: Hospital General Universitario de Alicante (HGUA). COVID19: Coronavirus disease 2019. ICU: Intensive care unit.
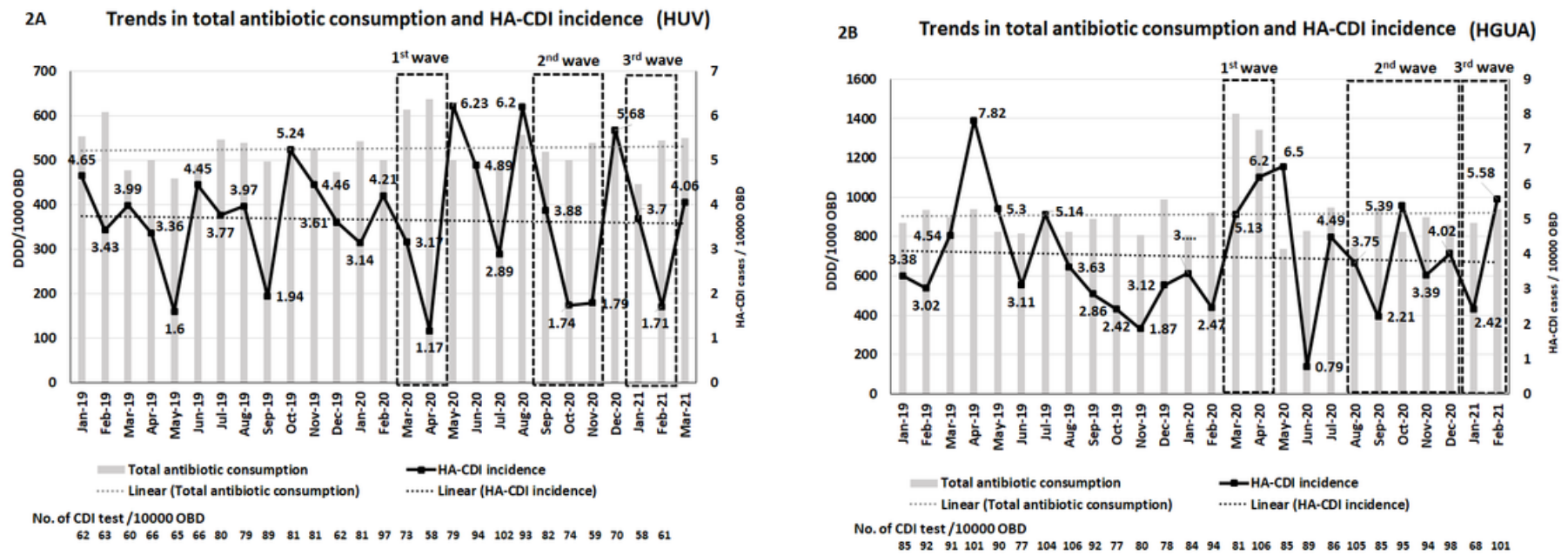

Figure 2

Trends in total antibiotic consumption and HA-CDI incidence during the study period. 2A: Hospital Universitario de Valme (HUV). 2B: Hospital General Universitario de Alicante (HGUA). DDD: Daily defined 
dose. HA-CDI: Health-care associated Clostridoides difficile infection. OBD: occupied bed-days. CDI: Clostridoides difficile infection.

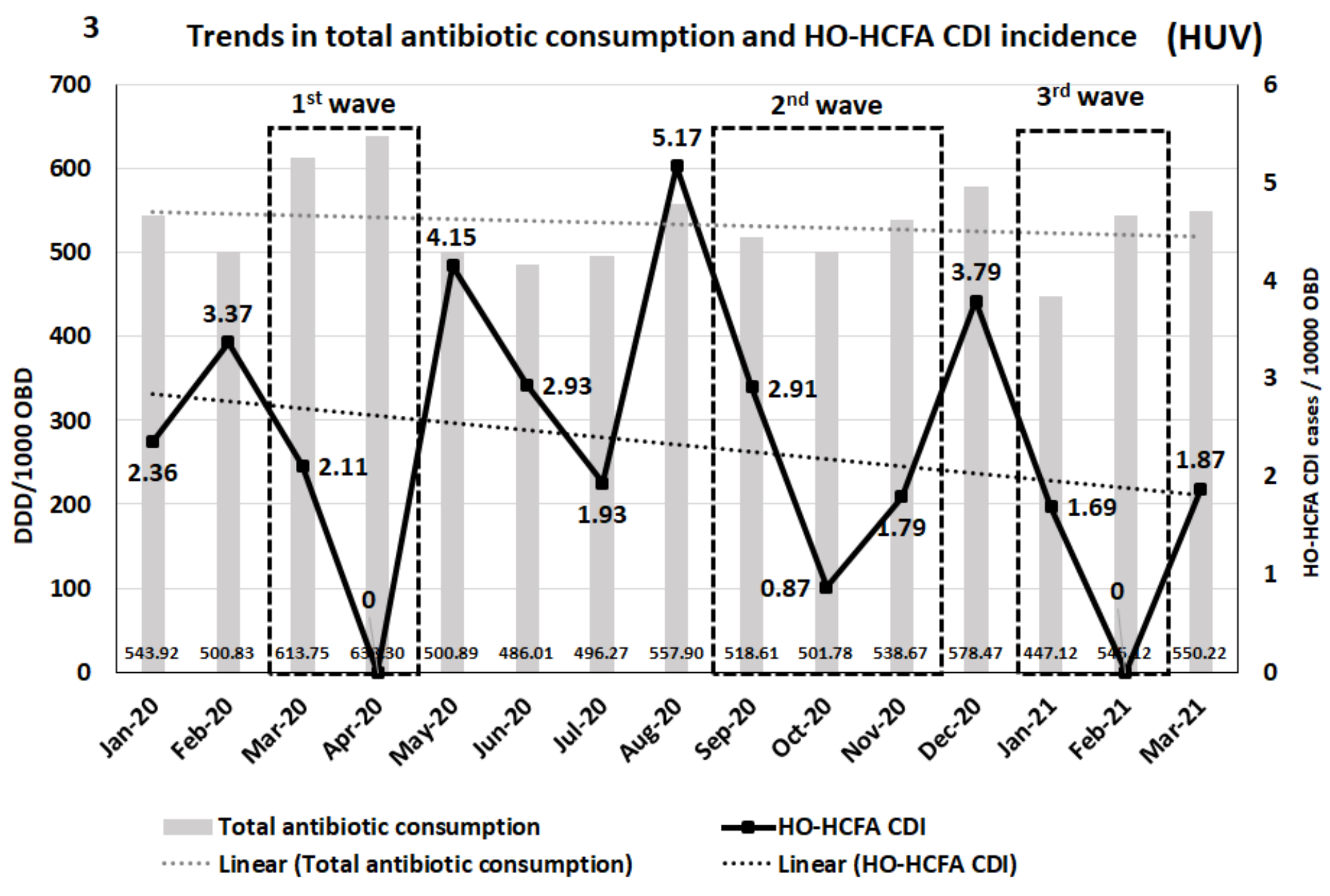

No. of CDI test / 10000 OBD

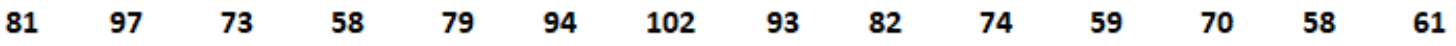

Figure 3

Trends in total antibiotic consumption and HO-HCFA incidence in Hospital Universitario de Valme during 2020-2021. DDD: Daily defined dose. HO-HCFA: Hospital-onset healthcare facility-associated Clostridoides difficile infection. OBD: occupied bed-days. CDI: Clostridoides difficile infection. 
$4 \mathrm{~A}$

Antibiotic consumption by period

Non COVID19 a COVID19

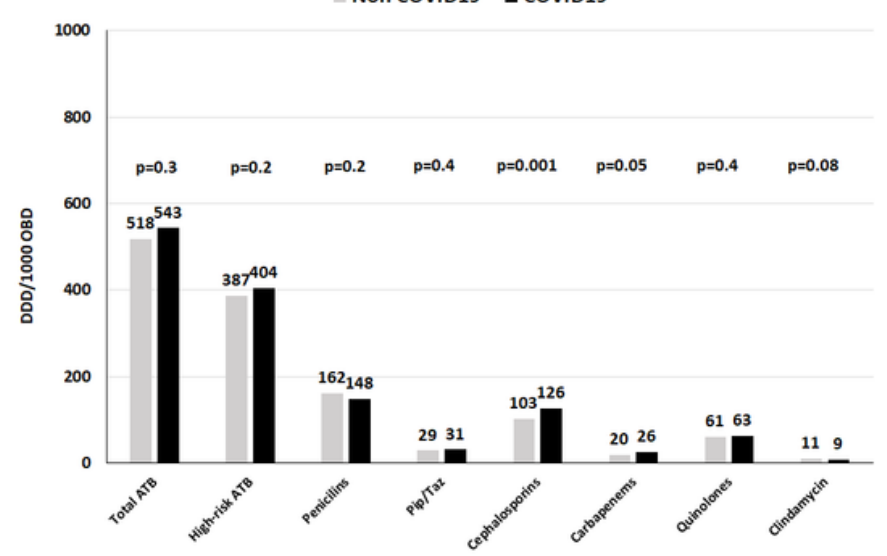

4B

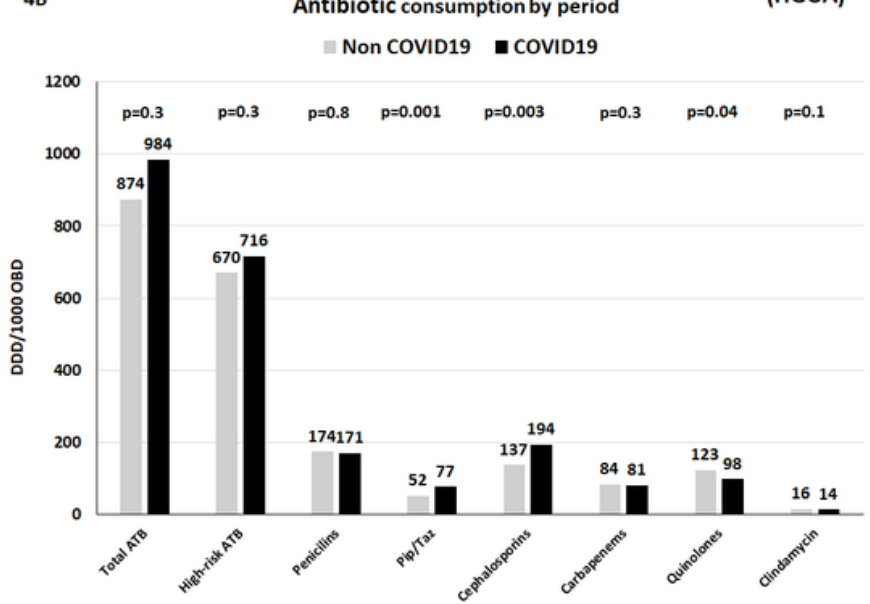

Figure 4

Antibiotic consumption by study period. 4A: Hospital Universitario de Valme (HUV). 4B: Hospital General Universitario de Alicante (HGUA). ATB: Antibiotics. COVID19: Coronavirus disease 2019. DDD: Daily defined dose. OBD: occupied bed-days. Pip/Taz: Piperacilin/Tazobactam. 